\title{
VDAC regulates AAC-mediated apoptosis and cytochrome $c$ release in yeast
}

\author{
Dário Trindade ${ }^{1,2}$, Clara Pereira ${ }^{3,4}$, Susana R. Chaves ${ }^{1}$, Stéphen Manon ${ }^{2}$, Manuela Côrte-Real ${ }^{1}$ and Maria \\ João Sousa ${ }^{1, *}$ \\ ${ }^{1}$ Centro de Biologia Molecular e Ambiental (CBMA), Departamento de Biologia, Universidade do Minho, Campus de Gualtar, 4710- \\ 057 Braga, Portugal. \\ ${ }^{2}$ Institut de Biochimie et de Génétique Cellulaires (IBGC), UMR5095 CNRS \& Université de Bordeaux, 1 Rue de Camille Saint-Saëns, \\ 33077 Bordeaux, France. \\ ${ }^{3}$ I3S-Instituto de Investigação e Inovação em Saúde, Universidade do Porto, Porto, 4200-135, Portugal. \\ ${ }^{4}$ IBMC-Institute for Molecular and Cell Biology, University of Porto, Porto, 4200-465, Portugal. \\ * Corresponding Author: \\ Maria João Sousa, Departamento de Biologia, Universidade do Minho, Campus de Gualtar; 4710-057 Braga, Portugal; Tel: +3 512536 \\ 01545; E-mail: mjsousa@bio.uminho.pt
}

\begin{abstract}
Mitochondrial outer membrane permeabilization is a key event in apoptosis processes leading to the release of lethal factors. We have previously shown that absence of the ADP/ATP carrier (AAC) proteins (yeast orthologues of mammalian ANT proteins) increased the resistance of yeast cells to acetic acid, preventing MOMP and the release of cytochrome $c$ from mitochondria during acetic acid - induced apoptosis. On the other hand, deletion of POR1 (yeast voltage-dependent anion channel - VDAC) increased the sensitivity of yeast cells to acetic acid. In the present work, we aimed to further characterize the role of yeast VDAC in acetic acid - induced apoptosis and assess if it functionally interacts with AAC proteins. We found that the sensitivity to acetic acid resulting from POR1 deletion is completely abrogated by the absence of AAC proteins, and propose that Por1p acts as a negative regulator of acetic acid - induced cell death by a mechanism dependent of AAC proteins, by acting on AAC - dependent cytochrome $c$ release. Moreover, we show that Por1p has a role in mitochondrial fusion that, contrary to its role in apoptosis, is not affected by the absence of AAC, and demonstrate that mitochondrial network fragmentation is not sufficient to induce release of cytochrome $c$ or sensitivity to acetic acid - induced apoptosis. This work enhances our understanding on cytochrome $c$ release during cell death, which may be relevant in pathological scenarios where MOMP is compromised.
\end{abstract}

doi: $10.15698 /$ mic2016.10.533 Received originally: 07.02.2016; in revised form: 23.07.2016, Accepted 27.07.2016, Published 25.08.2016.

Keywords: AAC, Por1, mitochondria, cytochrome c, acetic acid, Apoptosis.

\author{
Abbreviations: \\ $A A C-A D P / A T P$ carrier, \\ $A N T$ - adenine nucleotide translocator, \\ CyP-D - cyclophilin D, \\ Cyt c-cytochrome c, \\ IMM - inner mitochondrial membrane, \\ IMS - inter membrane space, \\ MOMP - mitochondrial outer \\ membrane permeabilization, \\ OMM - outer mitochondrial \\ membrane, \\ PTP - permeability transition pore, \\ VDAC - voltage dependent anion \\ channel.
}

\section{INTRODUCTION}

Mitochondrial outer membrane permeabilization (MOMP) is a key event in mammalian apoptosis processes leading to the release of lethal factors, like cytochrome $c$ (cyt $c$ ), apoptosis inducing factor (AIF) and Endonuclease G, which may activate downstream apoptotic and non-apoptotic death pathways. In mammalian cells, MOMP has been attributed to different mechanisms, namely: i) opening of the permeability transition pore (PTP), ii) formation of pores/channels in the outer mitochondrial membrane, either by Bcl-2 pro-apoptotic family members or ceramide molecules; and iii) interactions between the different pro- cesses and components [1-4]. However, the exact mechanisms of MOMP and its regulation remain to be clarified.

The permeability transition pore (PTP) is a pore formed at contact sites between the inner and outer mitochondrial membranes (IMM and OMM) [5] under conditions of elevated matrix $\mathrm{Ca}^{2+}$ concentrations, particularly when accompanied by oxidative stress and depletion of adenine nucleotides and $\mathrm{Mg}^{2+}[6,7]$. Long-lasting opening of the PTP results in the collapse of the electrochemical proton gradient, ROS accumulation and the equilibration of ionic gradients and solutes across the IMM, which eventually leads to 
osmotic swelling of the matrix, cristae remodelling and subsequent rupture of the OMM [8].

The exact molecular composition of the PTP is not completely defined and still remains a matter of debate, although it is generally accepted that PTP opening involves a multicomponent protein complex [9]. Several different proteins have been considered as either structural or regulatory components of the pore. Among the first recognized as participating in mitochondrial permeabilization were the voltage-dependent anion channel (VDAC) in the OMM $[10,11]$ and the adenine nucleotide translocator (ANT) in the IMM [12]. VDAC, also called mitochondrial porin, functions as a low-specificity molecular sieve and is considered responsible for the permeability of the OMM to several small molecules, therefore regulating the flow of metabolites between the cytoplasm and the mitochondrial inter membrane space (IMS) [13]. ANT is normally specific to the transport of adenine nucleotides, but a purified and functional ANT can also unselectively permeabilize lipid vesicles in the presence of $\mathrm{Ca}^{2+}$. Furthermore, $\mathrm{Ca}^{2+}$-induced mitochondrial permeability transition can be modulated by ligands of ANT $[6,14,15]$. These studies initially suggested that VDAC and ANT were constituents of the PTP, but genetic inactivation studies brought new insights into the molecular composition of the PTP. It is now proposed that PTP can result from the formation of a pore by the mitochondrial phosphate (Pi) carrier [16], from aggregation of misfolded and damaged membrane proteins [17], or be composed by dimers of the $F_{0} F_{1}$ ATP synthase [18]. Despite the multiple models, it is currently generally accepted that ANT and VDAC are non-essential components of the PTP but play important regulatory functions in the apoptotic process [19]. However, whether and how these two regulators functionally interact has not been elucidated yet.

Mitochondria of Saccharomyes cerevisiae are very similar to those from mammalian cells. In particular, this organism possesses three isoforms of the ADP/ATP carrier (AAC1, $A A C 2$ and $A A C 3$ ) that are orthologues of mammalian ANTs [20-22], as well as a porin (Por1p) that is an orthologue of mammalian VDACs, and a second porin homologue, Por $2 p$, which does not evidence channel properties $[23,24]$. A large-conductance unselective channel, having a size similar to the PTP, has also been detected in yeast mitochondria (YMUC). The ability of yeast mitochondria to undergo $\mathrm{Ca}^{2+}$-induced permeability transition suggests that the YMUC and mammalian PTP may be the expression of very similar events, originating the concept of "yeast PTP" [2527]. Though the exact composition of the YMUC remains to be elucidated, evidence argues against a contribution of AAC and/or porin $[28,29]$. Nevertheless, absence of Por1p is sufficient to alter the pore's voltage dependence and desensitizes it to $\mathrm{Ca}^{2+}$ regulation $[30,31]$. Furthermore, both AAC and Por1p have been implicated in yeast apoptosis induced by different stimuli. While the absence of AAC proteins increased the resistance of yeast cells to acetic acid and diamide (a thioloxidant compound that induces cyt $c$ release from mitochondria and cell death), preventing MOMP and the release of cyt $c$ from mitochondria during acetic acid - induced apoptosis [32], deletion of POR1 in- creased the sensitivity of yeast cells to acetic acid, hydrogen peroxide and diamide [32]. AAC proteins therefore seem to act as pro-death molecules and Por1p as a prosurvival protein. However, whether they share the same pathway in the regulation of yeast apoptosis remains to be clarified.

In this study, we sought to determine whether Por1p functionally interacts with AAC proteins, as well as its contribution to cyt $c$ release and yeast apoptosis induced by acetic acid treatment. We found that the sensitivity to acetic acid resulting from POR1 deletion is completely abrogated by the absence of AAC proteins, and a putative regulatory role of Por $1 p$ in cyt $c$ release from mitochondria depends on the presence of these IMM carriers. This indicates that Por1p may regulate cell survival by acting as a negative regulator of AAC proteins in the apoptotic cascade.

\section{RESULTS}

Absence of AAC proteins reverses the sensitivity of the $\Delta$ por1 mutant to acetic acid and diamide

We have previously shown that the increased resistance of $\Delta a a c 1 / 2 / 3$ cells to acetic acid is accompanied by a delay in the appearance of chromatin condensation, DNA strand breaks and loss of membrane integrity, contrasting with the early development of these events in $\Delta$ por1 cells. Additionally, the delay in the emergence of early and late apoptotic markers was associated with an impairment in MOMP and cyt $c$ release from mitochondria to the cytosol of acetic acid-treated yeast cells, which led to the conclusion that AAC proteins are required to promote cyt $c$ release to the cytosol, and that Por1p contributes to the resistance of yeast to apoptosis in this particular scenario [32]. In contrast, deletion of POR2 did not affect sensitivity to acetic acid - induced cell death (Fig. S1).

To study the interaction between AAC and Por1p in yeast apoptosis, the viability of wild type (wt), $\Delta$ por1, $\Delta a a c 1 / 2 / 3$ and $\Delta a a c 1 / 2 / 3 \Delta$ por 1 strains during acetic acid treatment $(180 \mathrm{mM})$ was evaluated by c.f.u. counting. Interestingly, the simultaneous absence of AAC and Por1p produces a resistance phenotype similar to that of the $\Delta a a c 1 / 2 / 3$ mutant, in contrast with the sensitivity phenotype exhibited by $\Delta$ por1 (Fig. $1 \mathrm{~A}$ ), as we had previously reported [32]. Indeed, $\Delta a a c 1 / 2 / 3$ and $\Delta a a c 1 / 2 / 3 \Delta$ por 1 strains revealed the highest plating efficiency after a 180 min exposure to acetic acid, exhibiting survival values (approximately $85 \%$ and $75 \%$, respectively) not significantly different from each other. On the other hand, the $\Delta$ por 1 strain displayed less than $10 \%$ survival after a $180 \mathrm{~min}$ treatment against approximately $40 \%$ of the wt (Fig. 1A), thus exhibiting increased sensitivity to acetic acid $(P<0.01)$. Furthermore, in the $\Delta a a c 1 / 2 / 3 \Delta$ por 1 mutant, the time course of cellular events associated with yeast apoptosis induced by acetic acid such as ROS production, chromatin condensation and loss of plasma membrane integrity observed in the $\Delta a a c 1 / 2 / 3 \Delta$ por1 mutant was identical to that previously observed for the $\Delta a a c 1 / 2 / 3$ strain (Fig. S2) [32]. A similar result was observed when $w t, \Delta a a c 1 / 2 / 3, \Delta$ por1 
A

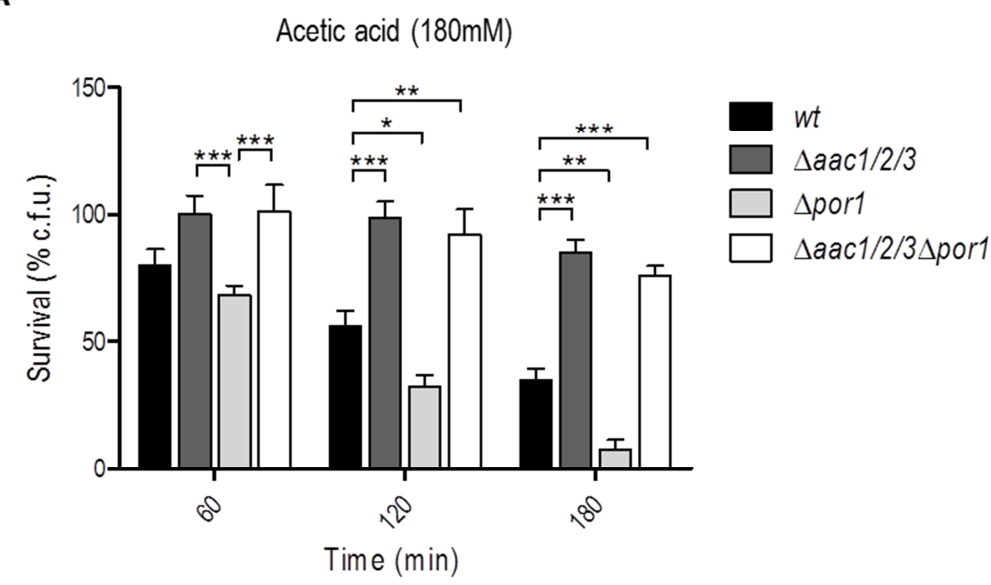

B

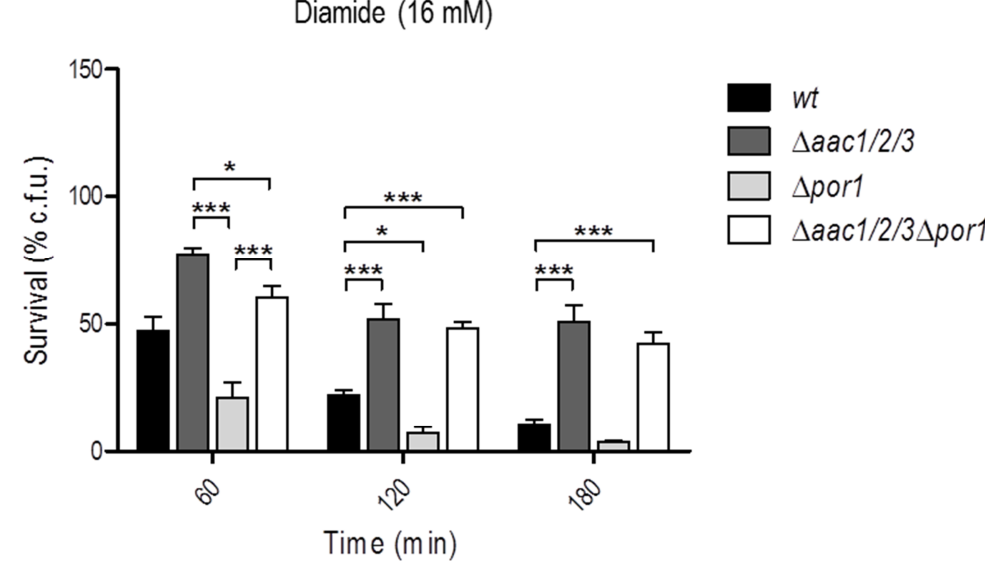

FIGURE 1: Absence of the AAC proteins promotes survival of $S$. cerevisiae to acetic acid and diamide reverting the sensitivity phenotype of $\Delta$ por1 cells. (A) Survival of $w t, \Delta a a c 1 / 2 / 3, \Delta$ por 1 and $\Delta a a c 1 / 2 / 3 \Delta$ por 1 cells after treatment with acetic acid (180 $\mathrm{mM}$ ) was determined by c.f.u. counts after 60, 120 and 180 minutes of treatment, considering the total c.f.u. number at T0 as $100 \%$ survival. (B) Survival of $w t, \Delta a a c 1 / 2 / 3$, $\Delta$ por1 and $\Delta a a c 1 / 2 / 3 \Delta$ por1 cells after treatment with diamide (16 mM) was determined by c.f.u. counts, as previously mentioned. Cells were precultured in YPD, and then grown O.N. in YPGal or YPD until an O.D.640nm of 1.5-2.0 was reached. Data represent mean \pm SEM of at least 3 independent experiments. Statistical analysis was performed using a Two-way ANOVA and Bonferroni posttests (P-values: $\left({ }^{*}\right) \mathrm{P}<0.05$; $\left({ }^{* *}\right) \mathrm{P}<0.01 ;\left({ }^{* * *}\right) \mathrm{P}<$ 0.001). and $\Delta a a c 1 / 2 / 3 \Delta$ por 1 cells were exposed to diamide (16 $\mathrm{mM}$ ) (Fig. 1B), a thioloxidant compound that induces cyt $c$ release from mitochondria and cell death $[32,33]$. Indeed, the absence of the AAC proteins increased the resistance of yeast cells to a lethal concentration of diamide, and reversed the sensitivity phenotype observed in $\Delta$ por 1 cells. Such observations suggest that the sensitivity to acetic acid - induced yeast apoptosis and to diamide - induced cell death resulting from Por1p deficiency, and thus the antiapoptotic role of Por1p, depends on the presence of AAC proteins. These results indicate there is a conserved regulatory system that depends on the interplay between AAC and Por1 proteins, and is capable of regulating the demise of yeast cells in different scenarios.

\section{Cytochrome $c$ release from mitochondria lacking Por1p is impaired in the absence of AAC}

Since the sensitivity of Por1p deficient cells to acetic acid was not observed in the absence of AAC proteins, the impact of POR1 deletion on AAC-mediated cyt $c$ release during acetic acid treatment was evaluated by redox spectrometry and Western blot of isolated mitochondria. While mitochondria from $\Delta$ por1 untreated cells produced stand- ard spectra, with an estimated cyt c/cyt $b$ ratio of 1.97 (Fig. $2 C)$ it was not possible to quantify this ratio in mitochondria prepared from $\Delta$ por1 cells exposed to $180 \mathrm{mM}$ acetic acid for $200 \mathrm{~min}$. Indeed, mitochondria integrity and isolation yield under this condition was significantly lower in comparison to the ones obtained with $w t, \Delta a a c 1 / 2 / 3$ and $\Delta a a c 1 / 2 / 3 \Delta$ por1 strains. To overcome this problem, the mitochondrial content of cytochromes $c+c_{1}$ and $b$ was quantified by redox spectrophotometry, in mitochondria isolated from $w t, \Delta p o r 1 \Delta a a c 1 / 2 / 3$, and $\Delta a a c 1 / 2 / 3 \Delta$ por 1 cells before and after 90 minutes of exposure to acetic acid. As expected, mitochondria from wt control cells showed cyt $c /$ cyt $b$ ratios of approximately 2.0 (Fig. $2 \mathrm{~A}$ ), while mitochondria from $\triangle a a c 1 / 2 / 3$ cells exhibited a slightly lower ratio (approximately 1.70 ). This observation has been previously reported and might be explained by the lower content of cyt $c$ in cells lacking the AAC proteins [32,34]. Interestingly, mitochondria from cells lacking both $A A C 1 / 2 / 3$ and Por1p exhibited a higher cyt $c / c y t b$ ratio than $w t$ mitochondria $(\approx 2.3)$. This could be in part explained by the lower amount of cyt $b$ detected in mitochondria from the $\Delta a a c 1 / 2 / 3 \Delta$ por1 mutant strain (not shown). While treatment with acetic acid led to a significant decrease in the 
cytochrome ratio of $\Delta$ por1 mitochondria, as it did in wt mitochondria, a much smaller variation was observed in $\Delta a a c 1 / 2 / 3$ mitochondria (Fig. 2B). Notably, the cyt $c /$ cyt $b$ ratio of $\Delta a a c 1 / 2 / 3 \Delta$ por1 isolated mitochondria was not altered (Fig. 2B), indicating that, like in $\Delta a a c 1 / 2 / 3$ [32], cyt $c$ release is severely impaired in $\Delta a a c 1 / 2 / 3 \Delta$ por 1 cells.

We confirmed these results by Western blot of mitochondrial samples, where we show a significant decrease of cyt $c$ content in mitochondria of acetic acid-treated $\Delta$ por1 cells (Fig. 3), as we previously described for the wt strain [32]. Indeed, absence of Por1p does not appear to compromise the release of cyt $c$, whose levels are decreased after treatment (approximately $25 \%$ less) in both $\Delta$ por1 and wt mitochondria (Fig. 3). On the other hand, cyt $c$ release from mitochondria of AAC-deficient cells is significantly impaired, and nearly all cyt $c$ (approximately 100\%) remains in mitochondria after acetic acid treatment (Fig. 3). Likewise, mitochondria from $\Delta a a c 1 / 2 / 3 \Delta$ por 1 cells seem to retain all of their cyt $c$, particularly if compared with mitochondria from treated wt or $\Delta$ por1 cells (Fig. 3). Taken to- gether, these observations suggest that, following acetic acid treatment, Por1p plays a role in cyt $c$ release in an AAC-dependent manner.

Por1p contributes to the mitochondrial tubular morphology independently of AAC proteins

The collapse of the mitochondrial network into small rounded mitochondria is a common phenomenon in many scenarios of apoptosis (for example [35], [36], reviewed in [37]), occurring alongside with MOMP [38]. Taking the above observations into account, we sought to assess whether the sensitive phenotype of $\Delta$ por 1 cells was related with increased mitochondrial fission, and hence a putative role of Por1p in mitochondrial morphology and its impact on the cellular response to acetic acid. To address this question, we expressed a mitochondria matrix-targeted GFP [39] in wt and $\Delta$ por1 strains, as well as in $\Delta$ por2 cells, deficient in the second orthologue of VDAC (BY4742, Euroscarf). In the parental strain, exponential phase cells exhibited mitochondria with a normal elongated tubular
A $w t$

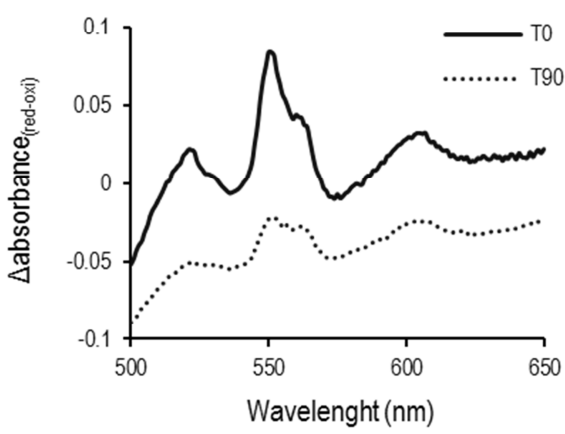

$\Delta$ por1

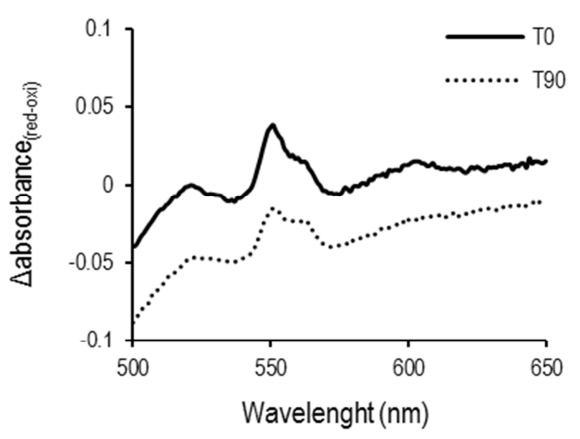

$\Delta$ aac1/2/3

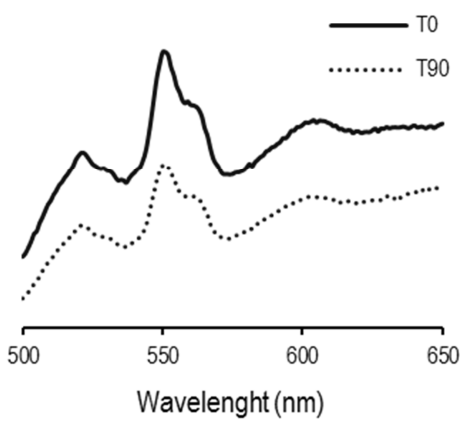

$\Delta$ aac1/2/3 $\Delta$ por1

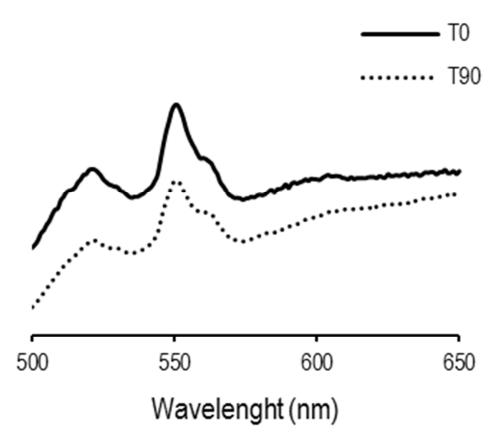

FIGURE 2: Mitochondria lacking the AAC proteins retain most of their cyt $c$ after acetic acid treatment. (A) Cells were precultured in YPD, and then grown O.N. in YPGal until an $\mathrm{OD}_{640 \mathrm{~nm}}$ of $1.5-2.0$ was reached, before adding acetic acid. Redox difference spectra of mitochondria isolated from $w t, \Delta a a c 1 / 2 / 3, \Delta p o r 1$ and $\Delta a a c 1 / 2 / 3 \Delta$ por1 control (full lines) or acetic acid-treated cells (dotted lines) are shown. Each graphic corresponds to one representative experiment from each tested strain. Peaks at $500 \mathrm{~nm}$ represent the amount of cytochromes $c+c 1$ while peaks at $561 \mathrm{~nm}$ represent the content of cytochrome $b$. (B) The corresponding cyt $c /$ cyt $b$ ratios estimated for $w t$, $\Delta a a c 1 / 2 / 3, \Delta$ por1 and $\Delta a a c 1 / 2 / 3 \Delta$ por 1 mitochondria extracted from control and acetic acid-treated ( $180 \mathrm{mM}, 90$ minutes) cells are represented in the lower table. Data represent mean \pm SD of at least 3 independent experiments.

\section{B}

\begin{tabular}{|c|c|c|c|c|}
\hline \multirow{2}{*}{ Cyt $c /$ Cyt $b$} & wt & $\Delta a a c 1 / 2 / 3$ & $\Delta p o r 1$ & $\Delta$ aac1/2/3 $\Delta$ por1 \\
\cline { 2 - 5 } T0 & $2.03 \pm 0.28$ & $1.71 \pm 0.07$ & $1.97 \pm 0.19$ & $2.33 \pm 0.05$ \\
T90 & $1.39 \pm 0.08$ & $1.55 \pm 0.30$ & $1.52 \pm 0.05$ & $2.30 \pm 0.55$ \\
\hline
\end{tabular}


network morphology (Fig. 4A). In the absence of Por1p, a high percentage of cells presented short spherical mitochondria of different sizes and often aggregated (Fig. 4A and $4 C$ ). Treatment of the wild-type cells with DIDS, a compound known to inhibit VDAC [40], led to a quick fragmentation of the mitochondrial network, which supports the view that VDAC contributes to mitochondrial fusion.

To assess whether POR1 deletion causes complete mitochondrial fragmentation or just fission/constriction of the inner membrane [41,42], mitochondria were stained with Mitotracker Red. This fluorescent probe is mitochondrion-selective and accumulates in response to mitochondrial membrane potential (negative in the matrix side). Mitotracker Red staining of $\Delta$ por 1 cells showed a high percentage of cells with fragmented morphology similar to that observed when using the mitochondrial matrix targeted-GFP. This observation indicates that an extensive mitochondrial fragmentation occurs in $\Delta$ por1 mutant mitochondria (Fig. 4A). In contrast, absence of Por2p led to an increase in the extent of the mitochondrial branching in comparison with the parental strain (Fig. 4A).

This effect however is not as dramatic as that observed in mutants impaired in mitochondrial fission, like the $\Delta d n m 1$ strain [43], suggesting that Por $2 p$ contributes to this process only to some extent. Quantification of the percentage of BY4742, $\Delta$ por 1 and $\Delta$ por 2 cells exhibiting fragmented morphology is shown in Fig. 4B. Since the absence of AAC proteins reverted the sensitive phenotype of $\Delta$ por1 mutant to acetic acid - induced apoptosis, we questioned whether it also reverted their fragmented mitochondrial morphology. In contrast with wt (W303-1B) and $\Delta a a c 1 / 2 / 3$ cells, $\Delta a a c 1 / 2 / 3 \Delta$ por 1 cells displayed the same fragmented mitochondrial morphology observed in the $\Delta$ por1 mutant, which suggests that the absence of AAC proteins has no effect in mitochondrial network organization (Fig. 4C). These observations suggest that, contrary to its role in apoptosis, the role of Por1p in mitochondrial fusion is not affected by the absence of AAC, and allows dissociating the fragmentation of the mitochondrial network from increased sensitivity to acetic acid and cyt $c$ release.

\section{DISCUSSION}

The mechanisms underlying mitochondrial membrane permeabilization and subsequent release of cyt $c$ in response to apoptotic stimuli have been the subject of extensive studies for the last decades. Throughout this period different components/mechanisms were postulated, including a proposed fundamental role of ANT and VDAC, which affect mitochondrial permeabilization. [44]. These proteins were originally thought to compose the scaffold structure of the PTP, but later genetic and molecular studies indicate they are not essential components of the PTP $[19,45]$, and their role in cell death is still poorly understood.

The yeast $S$. cerevisiae has played an important role towards the understanding of mitochondria permeabilization during apoptosis. Indeed, the yeast system has pro-

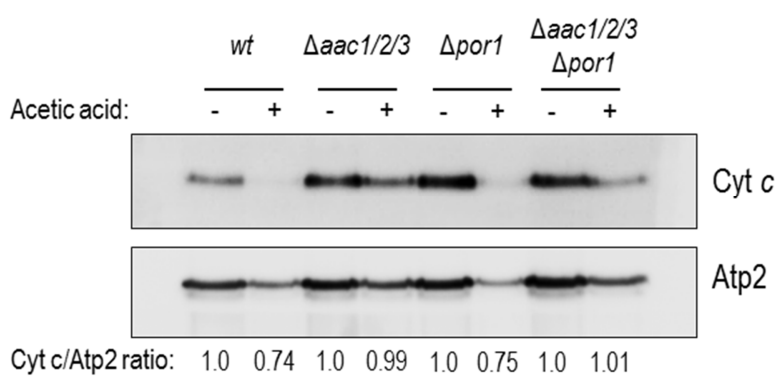

FIGURE 3: Cyt $c$ is released from $w t$ and $\Delta$ por1 mitochondria. Cells were pre-cultured in YPD, and then grown O.N. in YPGal until an $O_{640 \mathrm{~nm}}$ of 1.5-2.0 was reached, before adding acetic acid. One representative experiment of cyt $c$ immunodetection in $w t$, $\Delta a a c 1 / 2 / 3, \Delta$ por1, $\Delta a a c 1 / 2 / 3 \Delta$ por1 mitochondrial fractions, isolated from control and acetic-treated cells, is presented. The beta subunit of the $\mathrm{F} 1$ sector of mitochondrial $\mathrm{F}_{\mathrm{O}} \mathrm{F}_{1}$ ATP synthase (Atp2p) was used as control for the mitochondrial fractions. A densitometric analysis was performed (ImageJ software) and the corresponding cyt $c$ / Atp2 protein ratios are presented.

vided relevant insight into mechanisms of mitochondria permeabilization directly mediated by Bax, since it is devoid from obvious orthologues of the Bcl-2 family members, major regulators of mammalian apoptosis and MOMP, with the exception of Ybh3p [46], a non-canonical BH3-only protein [47-49] (for a review see [50]). Since yeast and mammalian mitochondria are similar and yeast contain orthologues of ANT proteins, porin, $F_{0} F_{1}$-ATP synthase and mitochondrial cyclophilin, which are putative components/regulators of the mammalian PTP, here, we further explore the yeast system to understand the involvement of these proteins in MOMP regulation and subsequent release of cyt $c$, independently of Bax and other Bcl-2 family proteins.

Like in the case of multicellular organisms, cyt $c$ release from mitochondria is a common event in several scenarios of yeast cell death, including deletion of the histone chaperone ASF1/CIA1, pheromone- and amiodarone-induced cell death, CDC48 mutation, and also $\mathrm{H}_{2} \mathrm{O}_{2}$ or acetic acid treatments [32,51-54] (for a review see [55]). We previously found that the release of cyt $c$ during acetic acid induced death of $S$. cerevisiae depends on the presence of the AAC proteins, an observation that correlates with the increased resistance of the $\Delta a a c 1 / 2 / 3$ mutant to this stress. We also identified Por1p as a key component of acetic acid - induced apoptosis, though with an opposite effect [32]. Here, we assessed the response of $S$. cerevisiae cells lacking the AAC proteins and Por1p to apoptosis-inducing concentrations of acetic acid, as well as their ability to release cyt $c$ from mitochondria. We found that yeast cells simultaneously depleted of AAC and Por1p exhibit an acetic acid-resistance phenotype, and cell survival identical to the $\Delta a a c 1 / 2 / 3$ strain. This effect is not exclusive to acetic acid, since the absence of AAC proteins can also protect yeast cells from death induced by diamide, a thioloxidant compound, as well as prevent the sensitivity phenotype result- 
A
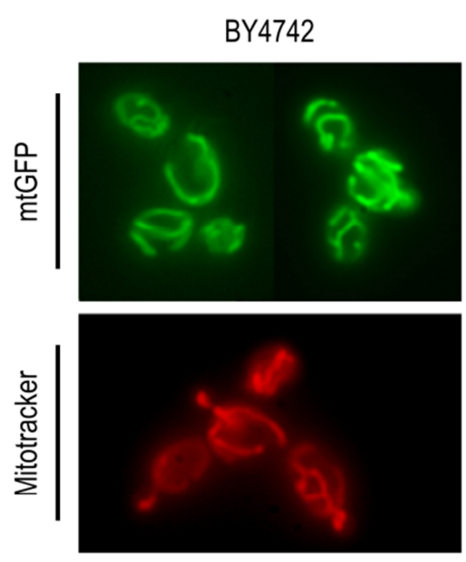

BY4742 + DIDS
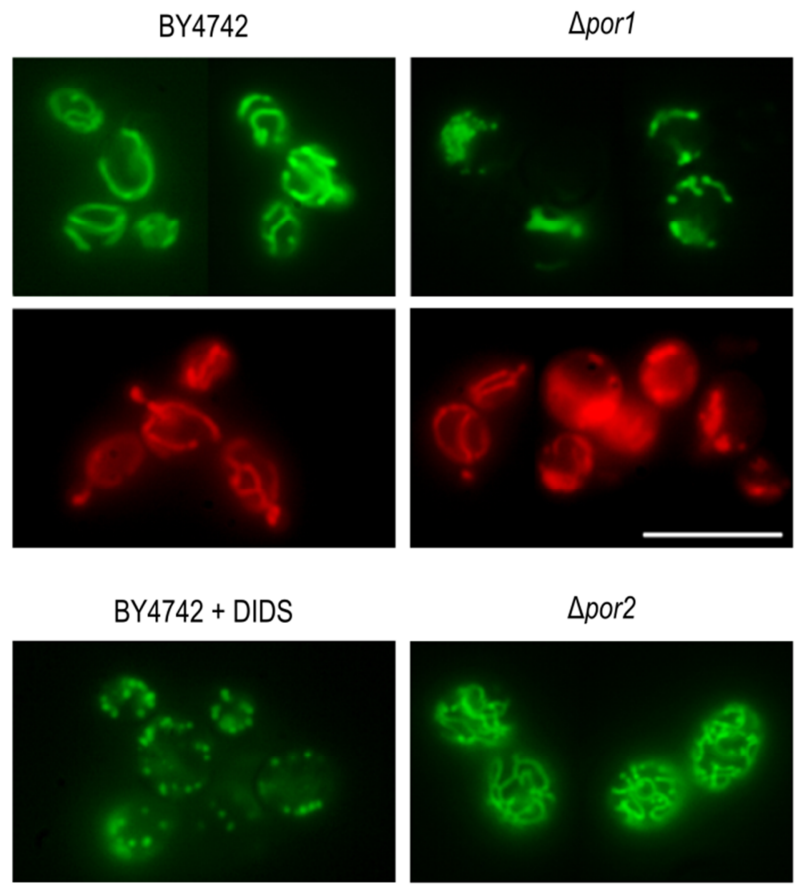

$\Delta$ por2
B

C

wt

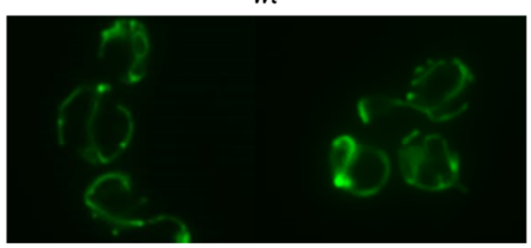

$\Delta a a c 1 / 2 / 3$

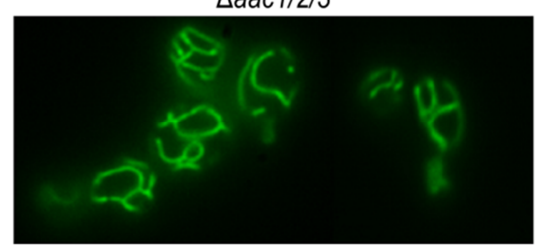

Fragmented/altered mitochondrial morphology (non-treated cells)

(\%)

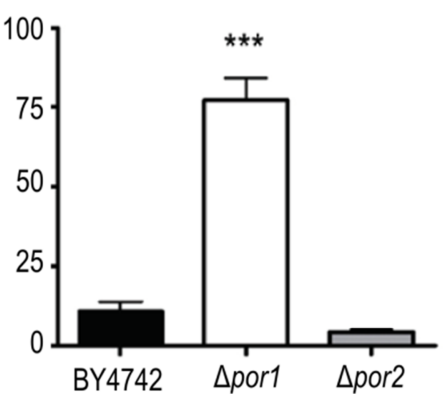

FIGURE 4: Por1p contributes to fusion in nontreated, healthy cells. (A) Mitochondrial morphology of BY4742, $\Delta p o r 1$ and $\Delta p o r 2$ cells, grown O.N. in SC Gal medium supplemented with the appropriate amino acids, was visualized by expression of a mitochondria matrixtargeted GFP. The BY4742 strain was treated for $5 \mathrm{~min}$ with $0.5 \mathrm{mM}$ of the anion channel inhibitor, DIDS. Mitochondrial morphology in BY4742 and $\Delta$ por1 strains was also visualized by Mitotracker Red straining. $B a r=10 \mu \mathrm{m}$. (B) Quantification of fragmented mitochondrial morphology (\%) for strains BY4742, $\Delta p o r 1$ and $\Delta p o r 2$ is shown. Values are means $\pm S D$ of four independent experiments. ${ }^{* * *} \mathrm{P}<0.001$, unpaired t-test. (C) Mitochondrial morphology in $\Delta a a c 1 / 2 / 3 \quad$ and $\Delta a a c 1 / 2 / 3 \Delta$ por1 strains expressing a matrix targeted GFP grown O.N. in SC Gal medium supplemented with the appropriate amino acids. Bar $=$ $10 \mu \mathrm{m}$.

ing from the absence of Por1p. We also show that deletion of Por1p does not affect cyt $c$ release in the $\Delta a a c 1 / 2 / 3$ background, since mitochondria from $\Delta a a c 1 / 2 / 3 \Delta$ por 1 cells, much like those from $\Delta a a c 1 / 2 / 3$ cells, retain most cyt $c$ after exposure to acetic acid. These observations corroborate the hypothesis that the AAC proteins are required for efficient cyt $c$ release, unlike Por1p which may play a distinct regulatory function, a scenario that has also been hypothesized in mammalian cells [9]. While AAC proteins have a significant impact on yeast cell survival in response to acetic acid, the role of Por1p seems to depend on the presence of the AACs. The fact that the resistance phenotype provided by the absence of AAC proteins actually overcomes the sensitivity phenotype resulting from POR1 deletion suggests that a regulatory role of Por1p would only be required when the AAC proteins are present in the IMM.

One possibility is that Por1p negatively regulates AAC proteins, either directly or by causing structural changes in mitochondria, counteracting AAC-mediated cyt $c$ release. Indeed, absence of Por1p results in increased difficulty to purify intact mitochondria only when AAC proteins are present, suggesting that Por1p can act on the AAC proteins to regulate mitochondria permeability. Although we did not observe differences in the extension of release of cyt $c$ in cells lacking Por1p, in comparison to the wt strain, the acetic acid sensitive phenotype of these cells and the lability of their mitochondria after acid treatment suggest that cyt $c$ release occurs earlier. Nevertheless, we cannot exclude the possibility that Por1p acts downstream of AAC but independently of it. 
Por1p is crucial to normal mitochondrial physiology associated with functions as diverse as the maintenance of redox state, mitochondrial DNA import and even cytoskeleton rearrangements [56-58]. Additionally, we present evidence supporting a role for Por1p in the organization of the mitochondrial network. This contribution of Por1p to mitochondrial morphology might be of outmost importance to understand the role of this protein in yeast apoptosis. In healthy growing cells, absence of Por1p leads to a phenotype of fragmentation of the mitochondrial network, while absence of Por2 $p$, a second VDAC isoform in yeast, leads to a slight increase in the extent of branching. Overexpression of $P O R 2$, a homolog of POR1, corrects the inability of $\triangle$ por 1 cells to grow on yeast media containing a non-fermentable carbon source at an elevated temperature $\left(37^{\circ} \mathrm{C}\right)$ [24]. However, no Por2p channels were detected electrophysiologically in reconstituted systems and its overexpression does not confer additional permeability to liposomes or intact mitochondria $[24,59]$. This evidence indicates that Por $2 p$, unlike Por $1 p$, is not a real porin and does not normally form channels. The different contributions of these proteins to mitochondria morphology further strengthen the idea that the two yeast VDAC isoforms have different, specialized functions.

Remodelling of the mitochondrial membrane has been suggested to play a role in the release of cyt $c$ in mammalian cells $[60,61]$. Accordingly, changes in mitochondria morphology dynamics might also modulate apoptosis in yeasts. Deletion of yeast fission proteins Dnm1p or Mvd1p delays the fragmentation of the mitochondrial network and, in case of cells lacking Mvd1p, promotes cell survival following a death stimulus or in ageing cells $[62,63]$. Ysp1p [53], Ysp2p [64] and Yca1p [65] are all required for fission of the mitochondrial network during apoptosis and their absence leads to apoptosis resistance. Mammalian proteins associated with the release of cyt $c$ were also shown to affect mitochondria morphology $[66,67]$, creating a possible link between apoptosis, cyt $c$ release and fragmentation of the mitochondrial network. We therefore hypothesised that the highly fragmented mitochondrial network in the $\Delta$ por1 strain could contribute to the increase susceptibility of this strain to apoptosis, for example by facilitating cyt $c$ release. Indeed, our data suggest that after 90 minutes of exposure to acetic acid, mitochondria from wt and $\Delta$ por 1 cells display a reduction in the level of cytochrome $c$. However, the fact that the $\Delta a a c 1 / 2 / 3 \Delta$ por $1 \mathrm{mu}-$ tant still displays a highly fragmented mitochondrial network but has an impaired release of cyt $c$ allows ruling out the fragmented phenotype as the cause of cyt $c$ release.

In mammalian cells, VDAC was implicated in the association of mitochondria with the cytoskeleton [68]. Disruption of the interaction of mitochondria with the cytoskeleton alters the normal mitochondrial morphology, giving origin to a fragmented mitochondrial network [69]. However, Blachly-Dyson and colleagues [24] reported that Por1p is not necessary for yeast mitochondrial segregation into the daughter cell, casting doubts on a role of Por1p in actin binding. As such, it will be important to assess if the destabilization of the mitochondrial network in the absence of Por1p is due to improper binding of mitochondria to the actin cytoskeleton or if Por1p is interfering with the organelle fission/fusion machinery.

In summary, we propose that Por1p acts as a negative regulator of cyt $c$ release from mitochondria of yeast cells exposed to acetic acid by counteracting AAC - dependent cyt $c$ release. Moreover, we show that Por1p has a role in mitochondrial morphology that, contrary to its role in apoptosis, is not affected by the absence of AAC, and demonstrate that mitochondrial network fragmentation is not sufficient to induce release of cyt $c$ or sensitivity to acetic acid - induced apoptosis.

This work enhances our understanding on cyt $c$ release, which may be relevant for mammalian cells, namely in pathological scenarios where MOMP is compromised, such as cancer [70].

\section{MATERIALS AND METHODS}

\section{Strains and Growth Conditions}

The yeast strains used in this study are listed in Table 1. Saccharomyces cerevisiae strains W303-1B and JL1-3 $\Delta 2 \Delta 3$, which is a derivate of W303 lacking the three isoforms of the AAC

TABLE 1. Yeast strains.

\begin{tabular}{|c|c|c|}
\hline Name & Genotype & Source/Reference \\
\hline$w t$ & W303-1B (MATa; ura3; trp1; leu2; his3; ade2; canR) & $\begin{array}{l}\text { Gift from Alexan- } \\
\text { der Tzagoloff }\end{array}$ \\
\hline$\Delta a a c 1 / 2 / 3$ & 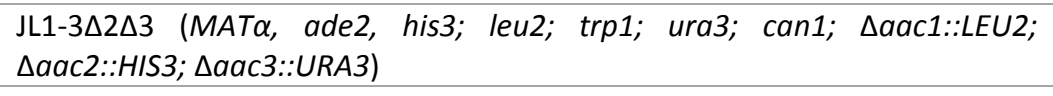 & Postis et al., 2005 \\
\hline$\Delta$ por1 & W303-1B; $\Delta$ por1::kanMX4 & This study \\
\hline$\Delta a a c 1 / 2 / 3 \Delta$ por1 & JL1-3 $22 \Delta 3 ; \Delta$ por1::kanMX4 & This study \\
\hline BY4742 wt & BY4742 (MATa ; his3; leu2; lys2; ura3) & Euroscarf \\
\hline BY4742 $\Delta$ por1 & BY4742 (MATa; his3; leu2; lys2; ura3; YNL055c::kanMX4/YNL055c) & Euroscarf \\
\hline BY4742 $\Delta$ por2 & 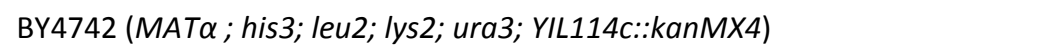 & Euroscarf \\
\hline
\end{tabular}


(AAC1, 2 and 3; [71]), were transformed with a $\triangle$ por1::kanMX4 interruption cassette, amplified by PCR from genomic DNA of BY4741 $\Delta$ por1 EUROSCARF deletion strain (EUROSCARF, Institute of Molecular Biosciences Johann Wolfgang Goethe-University Frankfurt, Germany), to generate $\Delta$ por 1 and $\Delta a a c 1 / 2 / 3 \Delta$ por 1 strains, respectively. Yeast cells were transformed by the lithium acetate method [72], selected in medium containing geneticin $(200 \mu \mathrm{g} / \mu \mathrm{L})$ and confirmed by PCR. For all experiments performed with these strains, cells were pre-grown in YPD medium ( $2 \%$ Glucose, $1 \%$ yeast extract, $1 \%$ bactopeptone), transferred to YPGal medium (2\% Galactose, $1 \%$ yeast extract, $1 \%$ bactopeptone) and incubated overnight (O.N.) at $30^{\circ} \mathrm{C}$ (200 r.p.m.) until an optical density of 1.5 2.0 was reached, essentially as previously described [32]. Strains BY4742 wt, BY4742 Lpor1 and BY4742 spor2 were obtained from the EUROSCARF's gene deletion library and transformed, along with $\triangle a a c 1 / 2 / 3$ and $\Delta a a c 1 / 2 / 3 \Delta$ por 1 , with plasmid YX232-mtGFP, containing the sequence of a mitochondria-targeted GFP [39]. These strains were grown in synthetic complete $(\mathrm{SC})$ medium $(0.67 \%$ Bacto yeast nitrogen base w/o amino acids, $2 \%(\mathrm{w} / \mathrm{v})$ Galactose, $0.2 \%(\mathrm{w} / \mathrm{v})$ Dropout mix) supplemented with the appropriate amino acids.

\section{Acetic acid and Diamide treatments}

For acetic acid tolerance and mitochondria extraction assays, acetic acid was added at a final concentration of $180 \mathrm{mM}$ to cultures grown O.N. until exponential growth phase $\left(\mathrm{OD}_{640 \mathrm{~nm}}=\right.$ 1.5-2.0) in YPGal medium. For diamide survival assays, a working solution of diazenedicarboxylic acid bis 5 $\mathrm{N}$,Ndimethylamide (diamide, from Sigma) was prepared in water (1 M), and added to cultures grown O.N. until exponential growth phase $\left(O D_{640 \mathrm{~nm}}=1.5-2.0\right)$ in YPD medium, to a final concentration of $16 \mathrm{mM}$. Viability of $w t, \Delta a a c 1 / 2 / 3, \Delta$ por1 and $\Delta a a c 1 / 2 / 3 \Delta$ por 1 cells during acetic acid and diamide treatments was evaluated by colony forming units (c.f.u.) counting. Samples collected at different time points during a 3 hours period were diluted, plated onto YPDA (YPD supplemented with $2 \%$ Agar; $200 \mu \mathrm{L}$ from a $1.25 \times 10^{3}$ cell/mL suspension) and grown for 2 days at $30^{\circ} \mathrm{C}$. Percentage of viable cells was estimated considering $100 \%$ survival the number of c.f.u. at time zero minutes, right before the addition of acetic acid.

\section{Mitochondria Isolation}

To isolate yeast mitochondria, approximately $5 \mathrm{~L}$ of $w t$, $\Delta a a c 1 / 2 / 3, \Delta$ por 1 and $\Delta a a c 1 / 2 / 3 \Delta$ por 1 cultures were grown O.N. in YPGal as previously described. Half of each culture was harvested and used as control (TO), while the remaining was subjected to acetic acid treatment $(180 \mathrm{mM})$ for 90 minutes. Control and acetic acid treated cells were converted into spheroplasts by enzymatic digestion with zymolyase (Zymolyase 20T, Seikagaku Biobusiness Corporation), disrupted by hand-potter or mechanical homogenization, and the mitochondrial fraction recovered after a series of differential centrifugations [73]. Mitochondrial suspensions were frozen in liquid nitrogen and stored at $-80^{\circ} \mathrm{C}$. All protein quantifications were performed by the Lowry method [74].

\section{Redox Spectrophotometry}

A mitochondrial suspension with the final concentration of 10 $\mathrm{mg} / \mathrm{mL}$ of mitochondria protein in recuperation buffer $(0.6 \mathrm{M}$ Mannitol; 10 mM Tris-maleate; 2 mM EGTA; pH 6.8) was prepared and equally divided into two eppendorf tubes. The ref- erence and sample tubes were oxidized and reduced with potassium ferricyanide and sodium dithionite, respectively. Sample absorbance was measured using a micro-plate spectrophotometer, and the redox difference spectra were acquired between 500 and $650 \mathrm{~nm}$. Cytochromes $c+c_{1}, b$ and $a+a_{3}$ were quantified by the OD differences, $550 \mathrm{~nm}$ minus $540 \mathrm{~nm}, 561 \mathrm{~nm}$ minus $575 \mathrm{~nm}$, and $603 \mathrm{~nm}$ minus $630 \mathrm{~nm}$, respectively.

\section{Western blot analysis}

For characterization of the mitochondrial fractions by Western blot, $50 \mu \mathrm{g}$ of proteins were precipitated with TCA, and solubilized in 2\% SDS before being separated by SDS-PAGE [75]. Proteins were then blotted onto PVDF membranes. Characterization was carried out with antibodies directed against cytochrome $c$ (rabbit polyclonal, 1:1000, custom-made by Millegen) and against the beta subunit of the F1 sector of mitochondrial $F_{0} F_{1}$ ATP synthase (rabbit polyclonal, 1:20000, home made by Jean Velours, IBGC, Bordeaux).

\section{Fluorescence microscopy}

Cells transformed with the plasmid YX232-mtGFP were grown O.N. as previously described, collected and immobilized in the slides by adding $0.5 \%(\mathrm{w} / \mathrm{v})$ agar prior to microscopy. When used, MitoTracker Red CMXRos (Molecular Probes) was added to the culture medium at a final concentration of $0.4 \mu \mathrm{g} / \mathrm{mL}$ and incubated for $20 \mathrm{~min}$ at $37^{\circ} \mathrm{C}$. For the assays with the anion channel inhibitor $4^{\prime}$-diisothiocyano-2,2'-disulfonic acid stilbene (DIDS), overnight grown cells were harvested and incubated in growth medium with $0.5 \mathrm{mM}$ of DIDS for $5 \mathrm{~min}$ [40]. Samples were analysed on a Leica Microsystems DM$5000 \mathrm{~B}$ epifluorescence microscope with appropriate filter settings using a $100 \times$ oil-immersion objective. Images were acquired with a Leica DCF350FX digital camera and processed with LAS AF Leica Microsystems software (Leica Mycrosystems).

\section{ACKNOWLEDGMENTS}

We would like to thank Doctor Alexander Tzagoloff, from Columbia University, Biological Sciences, New York, USA, for providing the yeast strain W303. D. Trindade was the recipient of a fellowship from Fundação Calouste Gulbenkian, Portugal. Work at the Institut de Biochimie et Génétique Cellulaires (IBGC), CNRS/Université Bordeaux Segalen was supported by Fundação Calouste Gulbenkian. This work was supported by FCT I.P through the strategic programme UID/BIA/04050/2013, project FCT-ANR/BEX-BCM/0175/2012 and a fellowship to $S$. Chaves (SFRH/ BPD/89980/2012).

\section{SUPPLEMENTAL MATERIAL}

All supplemental data for this article are available online at www.microbialcell.com.

\section{CONFLICT OF INTEREST}

The authors declare no conflict of interest.

\section{COPYRIGHT}

(C) 2016 Trindade et al. This is an open-access article released under the terms of the Creative Commons Attribution (CC BY) license, which allows the unrestricted use, 
distribution, and reproduction in any medium, provided the original author and source are acknowledged.

\section{REFERENCES}

1. Rasola A and Bernardi P (2007). The mitochondrial permeability transition pore and its involvement in cell death and in disease pathogenesis. Apoptosis Int J Program Cell Death 12(5): 815-833.

2. Zhivotovsky B, Galluzzi L, Kepp O, and Kroemer G (2009). Adenine nucleotide translocase: a component of the phylogenetically conserved cell death machinery. Cell Death Differ 16(11): 1419-1425.

3. Tsujimoto $Y$ and Shimizu $S$ (2007). Role of the mitochondrial membrane permeability transition in cell death. Apoptosis Int J Program Cell Death 12(5): 835-840.

4. Scharstuhl A, Mutsaers HAM, Pennings SWC, Russel FGM, and Wagener FADTG (2009). Involvement of VDAC, Bax and ceramides in the efflux of AIF from mitochondria during curcumin-induced apoptosis. Plos One 4(8): e6688.

5. Beutner G, Ruck A, Riede B, Welte W, and Brdiczka D (1996). Complexes between kinases, mitochondrial porin and adenylate translocator in rat brain resemble the permeability transition pore. FEBS Lett 396(2-3): 189-195.

6. Haworth RA and Hunter DR (1979). The Ca2+-induced membrane transition in mitochondria. II. Nature of the Ca2+ trigger site. Arch Biochem Biophys 195(2): 460-467.

7. Crompton $M$, Costi $A$, and Hayat $L$ (1987). Evidence for the presence of a reversible $\mathrm{Ca} 2+-$ dependent pore activated by oxidative stress in heart mitochondria. Biochem J 245(3): 915-918.

8. Bernardi P (1999). Mitochondrial transport of cations: channels, exchangers, and permeability transition. Physiol Rev 79(4): 11271155.

9. Siemen $D$ and Ziemer $M$ (2013). What is the nature of the mitochondrial permeability transition pore and what is it not? IUBMB Life 65(3): 255-262.

10. Szabó I and Zoratti M (1993). The mitochondrial permeability transition pore may comprise VDAC molecules. I. Binary structure and voltage dependence of the pore. FEBS Lett 330(2): 201-205.

11. Szabó I, De Pinto V, and Zoratti M (1993). The mitochondrial permeability transition pore may comprise VDAC molecules. II. The electrophysiological properties of VDAC are compatible with those of the mitochondrial megachannel. FEBS Lett 330(2): 206-210.

12. Halestrap AP and Davidson AM (1990). Inhibition of Ca2(+)induced large-amplitude swelling of liver and heart mitochondria by cyclosporin is probably caused by the inhibitor binding to mitochondrial-matrix peptidyl-prolyl cis-trans isomerase and preventing it interacting with the adenine nucleotide translocase. Biochem J 268(1): 153-160.

13. Colombini M (1979). A candidate for the permeability pathway of the outer mitochondrial membrane. Nature 279(5714): 643-645.

14. Hunter DR and Haworth RA (1979). The Ca2+-induced membrane transition in mitochondria. I. The protective mechanisms. Arch Biochem Biophys 195(2): 453-459.

15. Rück A, Dolder M, Wallimann T, and Brdiczka D (1998). Reconstituted adenine nucleotide translocase forms a channel for small molecules comparable to the mitochondrial permeability transition pore. FEBS Lett 426(1): 97-101.
Please cite this article as: Dário Trindade, Clara Pereira, Susana R. Chaves, Stéphen Manon, Manuela Côrte-Real and Maria João Sousa (2016). VDAC regulates AAC-mediated apoptosis and cytochrome $c$ release in yeast. Microbial Cell 3(10): 500-510. doi: 10.15698/mic2016.10.533

16. Leung AWC, Varanyuwatana $P$, and Halestrap AP (2008). The mitochondrial phosphate carrier interacts with cyclophilin $D$ and may play a key role in the permeability transition. J Biol Chem 283(39): 2631226323.

17. He L and Lemasters JJ (2002). Regulated and unregulated mitochondrial permeability transition pores: a new paradigm of pore structure and function? FEBS Lett 512(1-3): 1-7.

18. Giorgio V, von Stockum S, Antoniel M, Fabbro A, Fogolari F, Forte M, Glick GD, Petronilli V, Zoratti M, Szabó I, Lippe G, and Bernardi P (2013). Dimers of mitochondrial ATP synthase form the permeability transition pore. Proc Natl Acad Sci U S A 110(15): 5887-5892.

19. Kokoszka JE, Waymire KG, Levy SE, Sligh JE, Cai J, Jones DP, MacGregor GR, and Wallace DC (2004). The ADP/ATP translocator is not essential for the mitochondrial permeability transition pore. Nature 427(6973): 461-465.

20. O'Malley K, Pratt P, Robertson J, Lilly M, and Douglas MG (1982). Selection of the nuclear gene for the mitochondrial adenine nucleotide translocator by genetic complementation of the op1 mutation in yeast. J Biol Chem 257(4): 2097-2103.

21. Lawson JE and Douglas MG (1988). Separate genes encode functionally equivalent ADP/ATP carrier proteins in Saccharomyces cerevisiae. Isolation and analysis of AAC2. J Biol Chem 263(29): 1481214818.

22. Kolarov J, Kolarova N, and Nelson N (1990). A third ADP/ATP translocator gene in yeast. J Biol Chem 265(21): 12711-12716.

23. Mihara K and Sato R (1985). Molecular cloning and sequencing of CDNA for yeast porin, an outer mitochondrial membrane protein: a search for targeting signal in the primary structure. EMBO J 4(3): 769774.

24. Blachly-Dyson E, Song J, Wolfgang WJ, Colombini M, and Forte $M$ (1997). Multicopy suppressors of phenotypes resulting from the absence of yeast VDAC encode a VDAC-like protein. Mol Cell Biol 17(10): 5727-5738.

25. Jung DW, Bradshaw PC, and Pfeiffer DR (1997). Properties of a cyclosporin-insensitive permeability transition pore in yeast mitochondria. J Biol Chem 272(34): 21104-21112.

26. Yamada A, Yamamoto T, Yoshimura Y, Gouda S, Kawashima S, Yamazaki N, Yamashita K, Kataoka M, Nagata T, Terada H, Pfeiffer DR, and Shinohara $Y$ (2009). Ca2+-induced permeability transition can be observed even in yeast mitochondria under optimized experimental conditions. Biochim Biophys Acta 1787(12): 1486-1491.

27. Azzolin L, von Stockum S, Basso E, Petronilli V, Forte MA, and Bernardi $P$ (2010). The mitochondrial permeability transition from yeast to mammals. FEBS Lett 584(12): 2504-2509.

28. Ballarin C and Sorgato MC (1995). An electrophysiological study of yeast mitochondria. Evidence for two inner membrane anion channels sensitive to ATP. J Biol Chem 270(33): 19262-19268.

29. Lohret TA, Murphy RC, Drgoñ T, and Kinnally KW (1996). Activity of the mitochondrial multiple conductance channel is independent of the adenine nucleotide translocator. J Biol Chem 271(9): 4846-4849. 
30. Gutiérrez-Aguilar M, Pérez-Vázquez V, Bunoust $\mathrm{O}$, Manon S, Rigoulet $M$, and Uribe $S$ (2007). In yeast, $\mathrm{Ca}+$ and octylguanidine interact with porin (VDAC) preventing the mitochondrial permeability transition. Biochim Biophys Acta 1767(10): 1245-1251.

31. Lohret TA and Kinnally KW (1995). Multiple conductance channel activity of wild-type and voltage-dependent anion-selective channel (VDAC)-less yeast mitochondria. Biophys J 68(6): 2299-2309.

32. Pereira C, Camougrand N, Manon S, Sousa MJ, and Côrte-Real M (2007). ADP/ATP carrier is required for mitochondrial outer membrane permeabilization and cytochrome $\mathrm{c}$ release in yeast apoptosis. Mol Microbiol 66(3): 571-582.

33. Ueda S, Nakamura $H$, Masutani $H$, Sasada T, Yonehara S, Takabayashi A, Yamaoka Y, and Yodoi J (1998). Redox regulation of caspase-3(-like) protease activity: regulatory roles of thioredoxin and cytochrome c. J Immunol Baltim Md 1950 161(12): 6689-6695.

34. Gawaz M, Douglas MG, and Klingenberg M (1990). Structurefunction studies of adenine nucleotide transport in mitochondria. II. Biochemical analysis of distinct AAC1 and AAC2 proteins in yeast. J Biol Chem 265(24): 14202-14208.

35. Mancini M, Anderson BO, Caldwell E, Sedghinasab M, Paty PB, and Hockenbery DM (1997). Mitochondrial proliferation and paradoxical membrane depolarization during terminal differentiation and apoptosis in a human colon carcinoma cell line. J Cell Biol 138(2): 449-469.

36. Gao W, Pu Y, Luo KQ, and Chang DC (2001). Temporal relationship between cytochrome $c$ release and mitochondrial swelling during UVinduced apoptosis in living HeLa cells. J Cell Sci 114(Pt 15): 2855-2862.

37. Landes T and Martinou J-C (2011). Mitochondrial outer membrane permeabilization during apoptosis: the role of mitochondrial fission. Biochim Biophys Acta 1813(4): 540-545.

38. Martinou J-C and Youle RJ (2006). Which came first, the cytochrome c release or the mitochondrial fission? Cell Death Differ 13(8): 1291-1295.

39. Westermann B and Neupert W (2000). Mitochondria-targeted green fluorescent proteins: convenient tools for the study of organelle biogenesis in Saccharomyces cerevisiae. Yeast Chichester Engl 16(15): 1421-1427.

40. Shafir I, Feng W, and Shoshan-Barmataz V (1998). Voltagedependent anion channel proteins in synaptosomes of the torpedo electric organ: immunolocalization, purification, and characterization. J Bioenerg Biomembr 30(5): 499-510.

41. Jakobs S, Martini N, Schauss AC, Egner A, Westermann B, and Hell SW (2003). Spatial and temporal dynamics of budding yeast mitochondria lacking the division component Fis1p. J Cell Sci 116(Pt 10): 2005-2014.

42. Pevala V, Kolarov J, and Polcic P (2007). Alterations in mitochondrial morphology of Schizosaccharomyces pombe induced by celldeath promoting agents. Folia Microbiol (Praha) 52(4): 381-390.

43. Mozdy AD, McCaffery JM, and Shaw JM (2000). Dnm1p GTPasemediated mitochondrial fission is a multi-step process requiring the novel integral membrane component Fis1p. J Cell Biol 151(2): 367380

44. Kinnally KW, Peixoto PM, Ryu S-Y, and Dejean LM (2011). Is MPTP the gatekeeper for necrosis, apoptosis, or both? Biochim Biophys Acta 1813(4): 616-622.

45. Baines CP, Kaiser RA, Sheiko T, Craigen WJ, and Molkentin JD (2007). Voltage-dependent anion channels are dispensable for mitochondrial-dependent cell death. Nat Cell Biol 9(5): 550-555.
46. Büttner S, Ruli D, Vögtle F-N, Galluzzi L, Moitzi B, Eisenberg T, Kepp O, Habernig L, Carmona-Gutierrez D, Rockenfeller P, Laun P, Breitenbach $M$, Khoury C, Fröhlich K-U, Rechberger G, Meisinger C, Kroemer $\mathrm{G}$, and Madeo $\mathrm{F}$ (2011). A yeast $\mathrm{BH} 3$-only protein mediates the mitochondrial pathway of apoptosis. EMBO J 30(14): 2779-2792.

47. Ligr M, Madeo F, Fröhlich E, Hilt W, Fröhlich KU, and Wolf DH (1998). Mammalian Bax triggers apoptotic changes in yeast. FEBS Lett 438(1-2): 61-65.

48. Manon S, Chaudhuri B, and Guérin M (1997). Release of cytochrome $\mathrm{c}$ and decrease of cytochrome $\mathrm{c}$ oxidase in Bax-expressing yeast cells, and prevention of these effects by coexpression of $\mathrm{Bcl}-\mathrm{xL}$. FEBS Lett 415(1): 29-32.

49. Priault M, Camougrand N, Kinnally KW, Vallette FM, and Manon S (2003). Yeast as a tool to study Bax/mitochondrial interactions in cell death. FEMS Yeast Res 4(1): 15-27.

50. Renault TT, Dejean LM, and Manon S (2016). A brewing understanding of the regulation of Bax function by $\mathrm{Bcl}-\mathrm{xL}$ and $\mathrm{Bcl}-2$. Mech Ageing Dev

51. Braun RJ, Zischka $H$, Madeo $F$, Eisenberg $T$, Wissing $S$, Büttner $S$, Engelhardt SM, Büringer $D$, and Ueffing $M$ (2006). Crucial mitochondrial impairment upon CDC48 mutation in apoptotic yeast. J Biol Chem 281(35): 25757-25767.

52. Ludovico P, Rodrigues F, Almeida A, Silva MT, Barrientos A, and Côrte-Real M (2002). Cytochrome $c$ release and mitochondria involvement in programmed cell death induced by acetic acid in Saccharomyces cerevisiae. Mol Biol Cell 13(8): 2598-2606.

53. Pozniakovsky AI, Knorre DA, Markova OV, Hyman AA, Skulachev VP, and Severin FF (2005). Role of mitochondria in the pheromoneand amiodarone-induced programmed death of yeast. J Cell Biol 168(2): 257-269.

54. Yamaki M, Umehara T, Chimura T, and Horikoshi M (2001). Cell death with predominant apoptotic features in Saccharomyces cerevisiae mediated by deletion of the histone chaperone ASF1/CIA1. Genes Cells Devoted Mol Cell Mech 6(12): 1043-1054.

55. Pereira C, Silva RD, Saraiva L, Johansson B, Sousa MJ, and CôrteReal M (2008). Mitochondria-dependent apoptosis in yeast. Biochim Biophys Acta 1783(7): 1286-1302.

56. D'Souza-Schorey C, Boshans RL, McDonough M, Stahl PD, and Van Aelst $L$ (1997). A role for POR1, a Rac1-interacting protein, in ARF6mediated cytoskeletal rearrangements. EMBO J 16(17): 5445-5454.

57. Galganska H, Budzinska M, Wojtkowska M, and Kmita H (2008). Redox regulation of protein expression in Saccharomyces cerevisiae mitochondria: possible role of VDAC. Arch Biochem Biophys 479(1): 39-45.

58. Weber-Lotfi F, Ibrahim N, Boesch P, Cosset A, Konstantinov Y, Lightowlers RN, and Dietrich A (2009). Developing a genetic approach to investigate the mechanism of mitochondrial competence for DNA import. Biochim Biophys Acta 1787(5): 320-327.

59. Lee $A C, X u X, B l a c h l y-D y s o n ~ E$, Forte M, and Colombini M (1998). The role of yeast VDAC genes on the permeability of the mitochondrial outer membrane. J Membr Biol 161(2): 173-181.

60. Gazaryan IG and Brown AM (2007). Intersection between mitochondrial permeability pores and mitochondrial fusion/fission. Neurochem Res 32(4-5): 917-929.

61. Jeong S-Y and Seol D-W (2008). The role of mitochondria in apoptosis. BMB Rep 41(1): 11-22.

62. Fannjiang Y, Cheng W-C, Lee SJ, Qi B, Pevsner J, McCaffery JM, Hill RB, Basañez G, and Hardwick JM (2004). Mitochondrial fission proteins regulate programmed cell death in yeast. Genes Dev 18(22): 2785-2797. 
63. Scheckhuber CQ, Erjavec N, Tinazli A, Hamann A, Nyström T, and Osiewacz HD (2007). Reducing mitochondrial fission results in increased life span and fitness of two fungal ageing models. Nat Cell Biol 9(1): 99-105.

64. Sokolov S, Knorre D, Smirnova E, Markova O, Pozniakovsky A, Skulachev V, and Severin F (2006). Ysp2 mediates death of yeast induced by amiodarone or intracellular acidification. Biochim Biophys Acta 1757(9-10): 1366-1370.

65. Mazzoni C, Herker E, Palermo V, Jungwirth H, Eisenberg T, Madeo $F$, and Falcone $C$ (2005). Yeast caspase 1 links messenger RNA stability to apoptosis in yeast. EMBO Rep 6(11): 1076-1081.

66. Delivani P, Adrain C, Taylor RC, Duriez PJ, and Martin SJ (2006). Role for CED-9 and Egl-1 as regulators of mitochondrial fission and fusion dynamics. Mol Cell 21(6): 761-773.

67. Karbowski M, Norris KL, Cleland MM, Jeong S-Y, and Youle RJ (2006). Role of Bax and Bak in mitochondrial morphogenesis. Nature 443(7112): 658-662.

68. Lindén $M$ and Karlsson $G$ (1996). Identification of porin as a binding site for MAP2. Biochem Biophys Res Commun 218(3): 833-836.

69. Boldogh I, Vojtov N, Karmon S, and Pon LA (1998). Interaction between mitochondria and the actin cytoskeleton in budding yeast requires two integral mitochondrial outer membrane proteins, Mmm1p and Mdm10p. J Cell Biol 141(6): 1371-1381.
70. Rustin $P$ and Kroemer G (2007). Mitochondria and cancer. Ernst Schering Found Symp Proc (4): 1-21.

71. Postis V, De Marcos Lousa C, Arnou B, Lauquin GJ-M, and Trézéguet $V$ (2005). Subunits of the yeast mitochondrial ADP/ATP carrier: cooperation within the dimer. Biochemistry (Mosc) 44(45): 14732-14740.

72. Ito H, Fukuda $Y$, Murata $K$, and Kimura A (1983). Transformation of intact yeast cells treated with alkali cations. J Bacteriol 153(1): 163168.

73. Law RH, Manon S, Devenish RJ, and Nagley P (1995). ATP synthase from Saccharomyces cerevisiae. Methods Enzymol 260: 133-163.

74. Lowry OH, Rosebrough NJ, Farr AL, and Randall RJ (1951). Protein measurement with the Folin phenol reagent. J Biol Chem 193(1): 265275.

75. Laemmli UK (1970). Cleavage of structural proteins during the assembly of the head of bacteriophage T4. Nature 227(5259): 680685. 